\title{
Metal artifact reduction in computed tomography using local models in an image block-iterative scheme
}

\author{
Katrien Van Slambrouck ${ }^{1}$ and Johan Nuyts ${ }^{1}$ \\ Department of Nuclear Medicine, KU Leuven, Belgium and Medical Imaging Research Center (MIRC), KU Leuven, \\ Belgium
}

Purpose: In iterative reconstruction, metal artifacts can be reduced by applying more accurate reconstruction models that are usually also more computationally demanding. The hypothesis of this work is that these complex models only need to be applied in the vicinity of the metals and that a less complex model can be used for the remainder of the reconstruction volume.

\begin{abstract}
Method: A method is described that automatically divides the reconstruction volume into metal and nonmetal regions. The different regions are called patches. A different energy and resolution model can be assigned to each of the patches. The patches containing metals are reconstructed with a fully polychromatic spectral model (IMPACT) and if necessary with an increased resolution model. The patch without metals is reconstructed with a simple polychromatic model (MLTRC) that only includes the spectral behavior of water attenuation. Comparing the computational complexity of IMPACT and MLTRC gives a ratio of 8:3. The different patches are updated sequentially as in a grouped coordinate algorithm. Two phantoms were simulated and measured: a circular phantom containing small metal cylinders and a body phantom representing a human pelvis with two femoral implants. As a first test, the sequential update of the patches was applied while using the same energy model for all patches. Secondly, the local model approach was applied using MLTRC for non-metal regions and IMPACT for metal regions. The results of different iterative reconstruction schemes are compared to the results of projection completion, another important method for the reduction of metal artifacts.
\end{abstract}

Results: Reconstruction schemes including the sequential update of the patches result in images with less streak artifacts compared to a regular reconstruction. The sequential update of each of the metal regions improves the relative convergence of the metals (edges and attenuation values) against the rest of the image, which leads to an improved artifact reduction. Using the combined IMPACT+MLTRC model results in a similar image quality as using IMPACT everywhere, while providing an important benefit regarding computational complexity. Some streak and shadow artifacts were still present, but all structures present in the phantom could be observed. Projection completion results in reconstructions with less obvious streak and shadow artifacts but tends to deform or erase structures lying close to or in between metallic structures.

Conclusions: Metal artifact reduction with iterative reconstruction can be achieved by using complex models only locally without losing image quality. Separately updating metal regions leads to reduced streak artifacts. Structures lying close to or in between metals are often better reconstructed, compared to projection completion results, because all available information is used.

Keywords: metal artifact reduction, iterative reconstruction, CT, reconstruction, image artifacts

\section{INTRODUCTION}

Image quality of computed tomography (CT) reconstructions is often seriously degraded when metals are present in the field of view. Shadows and streaks mask the object reconstruction, which often hinders good interpretation of the results in the regions close to and in between metals.

Reconstruction for CT is typically done by filtered back projection (FBP). FBP is based on an analytical inversion of the Radon transform, yielding good and fast reconstructions in (nearly) ideal conditions. However, these conditions are not met when metals are present. Several metal artifact reduction (MAR) methods exist. Two important groups are projection completion (PC) and statistical reconstruction with advanced projection model.
In PC, the projections going through metals are considered as corrupted and are replaced by interpolated projections that cause less or no artifacts. PC starts by making an initial reconstruction, typically FBP. The metals in this reconstruction are segmented and forward projected. Hereupon, the metal projections in the measurement are removed and replaced by interpolated projections (e.g. linear interpolation ${ }^{1}$, polynomial interpolation $\left.{ }^{2-4}\right)$. The sinogram with the interpolated projections is then used for a new reconstruction. The metals are copied from the initial reconstruction and pasted into the final reconstruction. This method is fast, because usually based on FBP-like reconstruction and often yields artifact-free images. An important disadvantage of $\mathrm{PC}$ is the information loss caused by removing and interpolating projection data. At first, loss of information may be caused by the segmentation: the shape, edge regions, and attenuation values of the metals might 
be affected. When artifacts are severe, a proper segmentation is not always straightforward. In some applications as e.g. implant follow-up in orthopedic surgery, this information can be important. Moreover, by removing and replacing projections, not only information about the metals, but about all objects contributing to the removed projections is (partly) lost, especially edge information ${ }^{5}$. This causes new artifacts, even far away from the metals. The more projections that need to be replaced, the more information that is lost and the higher the chance for introducing new artifacts. Some more advanced PC methods try to compensate for this information loss. The information is retrieved by using tissue segmentation during interpolation ${ }^{4-7}$, by merging images $^{8,9}$ or projections ${ }^{10}$ or by using information out of other non-affected projections ${ }^{11,12}$.

An alternative method is iterative (statistical) reconstruction with an advanced projection model. Most artifacts arise because of incorrect or incomplete modeling of the acquisition. This implies that when a more accurate model is used, artifacts should be substantially reduced. Analytical methods as FBP use an inverse model, which makes adaptations to the model very difficult. Iterative methods make use of a forward model which is more suitable for the introduction of more complex models.

A more elaborated discussion on the origin of (metal) artifacts can be found in Barrett and Neat ${ }^{13}$ and De Man et al. ${ }^{14}$. The most important causes of metal artifacts are: beam hardening, (non)-linear partial volume effects, scatter, noise, and motion. Beam hardening is due to the polychromatic nature of X-rays emerging from the tube causing a non-linear behavior between absorber thickness and linear attenuation. Classical algorithms assume a monochromatic X-ray beam and consequently a linear behavior. This leads to cupping artifacts and dark shadows and streaks in between and around the metals. Non-linear partial volume artifacts stem from integrating the attenuation over the finite width of the X-ray beam. Most algorithms implicitly assume this results in a simple averaging of the attenuation. Actually, it yields the (log-converted) intensity average. Ignoring this effect gives rise to dark and white streaks connecting the edges of highly attenuation objects, like metals. Scatter is secondary radiation coming from electron-photon interactions (Compton scatter) or atom-photon interactions (Rayleigh scatter). This secondary radiation contaminates the measurement. Usually scattered photons are ignored. Artifacts arising here are also cupping, shadows and streaks. Another source of contamination is noise, which has several origins. The quantum nature of the photons is considered as the most important. In the presence of metals this noise can lead to fine streaks connecting the metals. Motion will also lead to severe artifacts in the presence of metals but will not be considered in this study.

Iterative reconstruction with maximization of the Poisson likelihood (maximum likelihood (ML) reconstruction), inherently takes into account a Poisson model for noise in the projection data. In most of current CT systems, energy-integrating detectors are used, which results in a compound Poisson distribution of the signal. However, simple Poisson is usually a sufficient approximation $^{15}$. ML reconstruction usually leads to reduced noise, compared to FBP, in the reconstructed image. The acquisition model can be adapted to include the effects described above that cause severe artifacts in the presence of metals. Several maximum likelihood iterative algorithms exist that have a polychromatic model to reduce beam hardening artifacts ${ }^{16-19}$. In De $\operatorname{Man}^{20}$ and Zbijewski and Beekman ${ }^{21}$ ML methods reducing (non)linear partial volume artifacts are presented. Further, if an estimate of the scatter contribution is available, inclusion in the model is straightforward. Using more accurate models strongly reduces artifacts. The main disadvantage of all iterative methods is the increased computation time. Unfortunately, when the complexity of the model increases, the computation time is enormously prolonged and this makes use in daily practice almost impossible.

In this paper we present a framework for iterative maximum likelihood reconstruction that limits the extra time caused by increasing the model complexity. Complex models are only necessary in certain regions of the object, more specifically around metals, and less complex models can be used for the remainder of the object. A patchwork structure is presented which allows local model reconstruction. Different projection models will be used in different parts of the reconstruction volume. Using complex models where needed and simpler models elsewhere should limit the computation time while keeping similar image quality. Secondly, an improved artifact reduction can be achieved by altering the convergence of the different image parts. The results of the local model iterative reconstruction schemes will be compared with FBP-based projection completions methods.

\section{METHODS}

In this section the different steps in making a local model reconstruction are explained. The first part explains how the different regions are defined. Secondly, the different models that will be used are explained. Finally, the combination of models is described. At the end of this section, the projection completion methods used as comparison, are also briefly described.

\section{A. Patch definition}

The patchwork structure divides the reconstruction volume in non-overlapping regions, patches, and assigns particular reconstruction parameters to each of these regions. The patches are defined based upon segmentation of an initial reconstruction. This reconstruction can be made by FBP, but a single iteration of an iterative method is more desirable because then less artifacts are 
present which simplifies the segmentation. As the shape of the segmentation will not determine the final shape of the object, the segmentation accuracy is not crucial, although it should preferentially contain the whole metal. In this study the metal regions are defined by a thresholding method. These regions are dilated with a few pixels to ensure inclusion of the entire metal, see figure 1. Each of the metal regions is considered a patch, the remainder of the object is another patch. An object with four metallic inserts, thus results in five patches. Note that thresholding segments the metals each in a separate patch. This means that bone structure would be assigned to the major patch together with soft tissue and air.

\section{B. Maximum Likelihood Reconstruction and the different projection models}

The reconstruction is done by a maximum likelihood method, which is based on the Poisson likelihood:

$$
L=\sum_{i}\left(y_{i} \ln \hat{y}_{i}-\hat{y}_{i}\right)
$$

with $i$ the index of the projection lines, $y_{i}$ the measured transmission scan and $\hat{y}_{i}$ the estimated transmission scan, computed from the current reconstruction image $\vec{\mu}=\left\{\mu_{j}\right\}$, with $\mu_{j}$ the linear attenuation coefficient in voxel $j$. A gradient ascent algorithm for optimizing this likelihood, as a function of $\vec{\mu}$, has been proposed before by Nuyts et al. ${ }^{22}$ and Fessler et al. ${ }^{23}$. The algorithm can be derived as follows: starting with a quadratic expansion and the condition that $\frac{\partial^{2} L}{\partial \mu_{j} \partial \mu_{h}} \leq 0$ for all $j$ and $h$ (negative definite Hessian):

$$
\begin{gathered}
L(\vec{\mu}+\Delta \vec{\mu}) \simeq T_{1}(\vec{\mu} ; \Delta \vec{\mu}) \\
=L(\vec{\mu})+\left.\sum_{j} \frac{\partial L}{\partial \mu_{j}}\right|_{\vec{\mu}} \Delta \mu_{j}+\left.\frac{1}{2} \sum_{j, h} \frac{\partial^{2} L}{\partial \mu_{j} \partial \mu_{h}}\right|_{\vec{\mu}} \Delta \mu_{j} \Delta \mu_{h}(3) \\
\geq L(\vec{\mu})+\left.\sum_{j} \frac{\partial L}{\partial \mu_{j}}\right|_{\vec{\mu}} \Delta \mu_{j}+\left.\frac{1}{2} \sum_{j, h} \frac{\partial^{2} L}{\partial \mu_{j} \partial \mu_{h}}\right|_{\vec{\mu}} \Delta \mu_{j}{ }^{2} .
\end{gathered}
$$

The last expression (4) follows from $2\left(\mu_{j}-\mu_{j}^{n}\right)\left(\mu_{h}-\right.$ $\left.\mu_{h}^{n}\right) \leq\left(\mu_{j}-\mu_{j}^{n}\right)^{2}+\left(\mu_{h}-\mu_{h}^{n}\right)^{2}$ and the fact that the second derivatives are negative. It is a surrogate function for $T_{1}(\vec{\mu} ; \Delta \vec{\mu})(3)$, because it is equal to $T_{1}$ at $\Delta \mu=0$ and below $T_{1}$ elsewhere. Maximization of this surrogate leads to the following update for $\Delta \mu_{j}$ :

$$
\Delta \mu_{j}=-\frac{\frac{\partial L}{\partial \mu_{j}}}{\sum_{h} \frac{\partial^{2} L}{\partial \mu_{j} \partial \mu_{h}}},
$$

where all derivatives are evaluated in the current reconstruction $\vec{\mu}$. To calculate the update for $\mu_{j}$, a model for $\hat{y}_{i}$ needs to be defined. In this work the energy and resolution properties of the model can be changed. Furthermore a scatter term can be included.

\section{Energy model}

a. Monochromatic model: MLTR The monochromatic model is the one used in MLTR (ML for transmission $)^{22}$ :

$$
\hat{y}_{i}=b_{i} \exp \left(-\sum_{j} l_{i j} \mu_{j}\right)+s_{i}
$$

where $b_{i}$ is the blank value for projection line $i$ in the presence of (bowtie) filters, $l_{i j}$ is the intersection length of projection line $i$ and pixel $j$, and $s_{i}$ is the scatter term for projection ray $i$. This yields the following update step:

$$
\Delta \mu_{j}=\frac{\sum_{i} l_{i j} \frac{\hat{y}_{i}-s_{i}}{\hat{y}_{i}}\left(\hat{y}_{i}-y_{i}\right)}{\sum_{i} l_{i j} \sum_{h} l_{i h}\left(\hat{y}_{i}-s_{i}\right)\left(1-\frac{y_{i} s_{i}}{\hat{y}_{i}^{2}}\right)} .
$$

A complete update step requires one projection and two backprojections.

b. Polychromatic water model: MLTRC MLTRC is a polychromatic extension of MLTR which takes into account the polychromatic behavior of the X-rays going through water. The projection model becomes:

$$
\begin{gathered}
\hat{y}_{i}=\sum_{k} \hat{y}_{i k}+s_{i}=\sum_{k} b_{i k} \exp \left(-P_{k} \sum_{j} l_{i j} \mu_{j, \mathrm{ref}}\right)+s_{i}, \\
P_{k}=\frac{\mu_{k}^{\text {water }}}{\mu_{\text {ref }}^{\text {water }}},
\end{gathered}
$$

where $k$ is the energy bin and $b_{i k}$ the blank value for projection line $i$ at energy $k$ in the presence of (bowtie) filters, $\mu_{\text {ref }}^{\text {water }}$ is the linear attenuation coefficient of water at a chosen reference energy and $\mu_{j, \text { ref }}$ is the reconstructed linear attenuation coefficient in pixel $j$ at the same reference energy. The MLTRC update becomes:

$$
\Delta \mu_{j}=\frac{\sum_{i} l_{i j} Y_{i}^{P}\left(1-\frac{y_{i}}{\hat{y}_{i}}\right)}{\sum_{i} l_{i j} \sum_{h} l_{i h}\left[\left(1-\frac{y_{i}}{\hat{y}_{i}}\right) Y_{i}^{P P}+\frac{y_{i}}{\hat{y}_{i}^{2}}\left(Y_{i}^{P}\right)^{2}\right]}
$$

with

$$
\begin{aligned}
Y_{i}^{P} & =\sum_{k} P_{k} \hat{y}_{i k} \\
Y_{i}^{P P} & =\sum_{k}\left(P_{k}\right)^{2} \hat{y}_{i k} .
\end{aligned}
$$

Usually a limited set of energy bins, e.g. 10, is enough to represent the spectrum. Note that the complexity (in terms of (back)projections) of this model is the same as that of regular MLTR. 

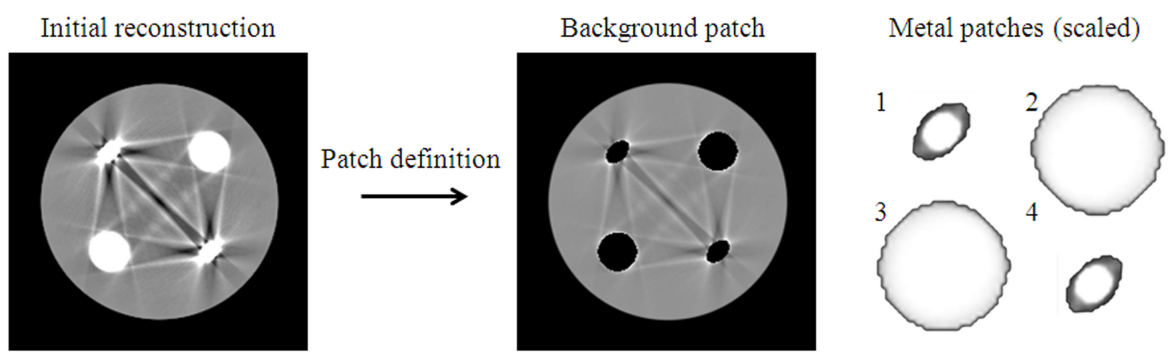

FIG. 1. Patch definition Definition of the different patches based upon segmentation of an initial reconstruction (1 iteration with 116 subsets of IMPACT). Result: a background patch and four metal patches.
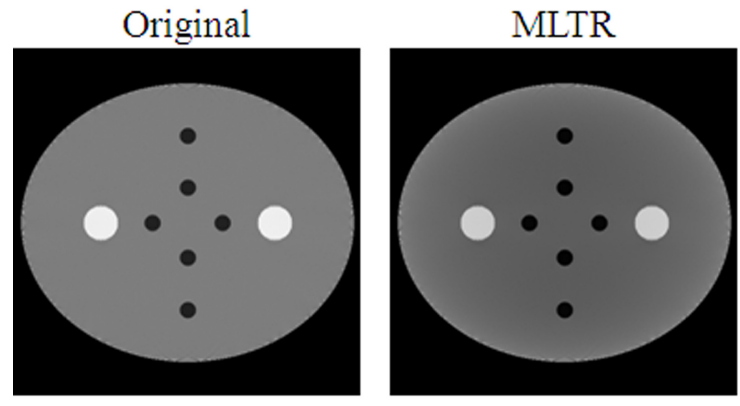

MLTRC

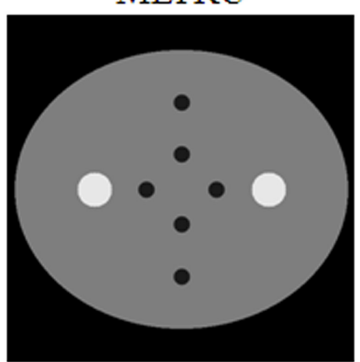

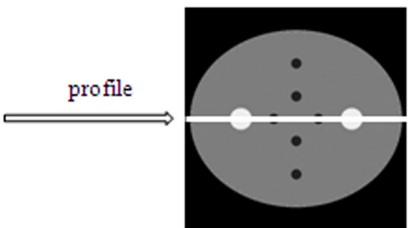

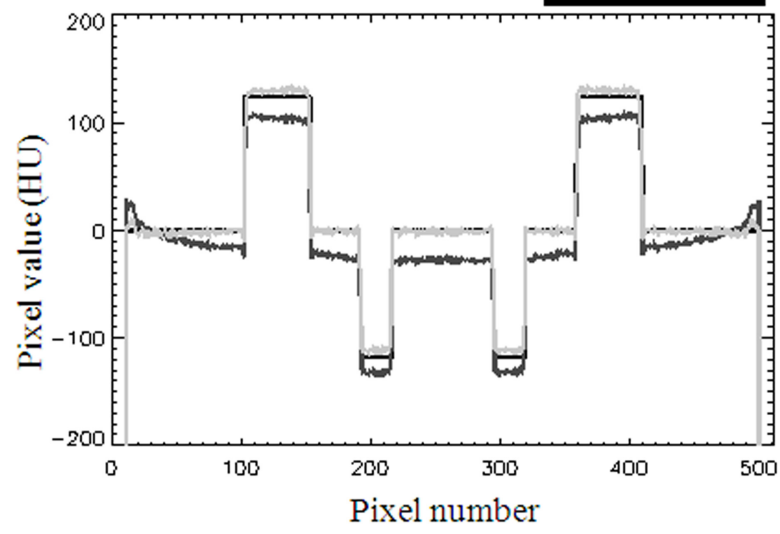

FIG. 2. MLTR and MLTRC Reconstruction of (computer) water phantom with fat (darker) and PMMA (brighter) inserts. Reconstructions are performed for 10 iterations with 116 subsets with MLTR and MLTRC. (Window $=300$ HU, Center $=0$ $\mathrm{HU})$. The profile through the reconstruction is compared with the true profile, where black $=$ original, dark gray $=$ MLTR and light gray $=$ MLTRC.

The effect of using MLTRC compared to MLTR is shown in the next example. A simple polychromatic simulation (spectrum of $120 \mathrm{kV}$ ) is performed using a phantom of $50 \mathrm{~cm}$ with several 'fat' and PMMA (polymethyl methacrylate) details. The results for a reconstruction with MLTR and MLTRC are shown in figure 2. For MLTR the typical cupping is present, which is eliminated using MLTRC. Materials other than water are only partly corrected. The more the spectral properties of a material differ from those of water, the less effective this correction will be. In fact, the effect of using MLTRC is similar to using a (polynomial) water pre-correction on raw data ${ }^{24}$. However, with MLTRC projections for different energies can be calculated which will be necessary when combining it with fully polychromatic models.

c. Full polychromatic model: IMPACT The IMPACT (iterative maximum likelihood polychromatic algorithm for $\mathrm{CT})^{16}$ projection model uses a fully poly- chromatic projection estimate:

$$
\begin{aligned}
\hat{y}_{i} & =\sum_{k} \hat{y}_{i k}+s_{i} \\
& =\sum_{k} b_{i k} \exp \left(-\Theta_{k} \sum_{j} l_{i j} \theta\left(\mu_{j, \mathrm{ref}}\right)-\Phi_{k} \sum_{j} l_{i j} \phi\left(\mu_{j, \mathrm{ref}}\right)\right)
\end{aligned}
$$

The energy dependent linear attenuation is written as a linear combination of the Compton and the photo-electric component, with $\Theta_{k}$ and $\Phi_{k}$ the energy dependence of respectively Compton scattering and photo-electric effect, and $\vec{\theta}$ and $\vec{\phi}$ the material dependence. We assume that $\vec{\theta}$ and $\vec{\phi}$ are unambiguously determined by the attenuation at a chosen reference energy: $\theta_{j}=\theta\left(\mu_{j, \text { ref }}\right)$ and $\phi_{j}=\phi\left(\mu_{j, \mathrm{ref}}\right)$. To obtain this, we assume that a list of possible materials is available, which has been sorted according to increasing effective attenuation. We further assume that attenuation in every pixel can be well mod- 
eled as a mixture of two adjacent materials in this list. The update becomes:

$$
\Delta \mu_{j}=\frac{\phi_{j}^{\prime} \sum_{i} l_{i j} e_{i} Y_{i}^{\Phi}+\theta_{j}^{\prime} \sum_{i} l_{i j} e_{i} Y_{i}^{\Theta}}{\phi_{j}^{\prime} \sum_{i} l_{i j} M_{i}+\theta_{j}^{\prime} \sum_{i} l_{i j} N_{i}}
$$

with

$$
\begin{array}{rlr}
\phi_{j}^{\prime} & =\frac{\partial \phi}{\partial \mu_{j}} \quad \theta_{j}^{\prime}=\frac{\partial \theta}{\partial \mu_{j}} \\
e_{i} & =1-\frac{y_{i}}{\hat{y}_{i}} & Y_{i}^{\Theta}=\sum_{k} \Theta_{k} \hat{y}_{i k} \\
Y_{i}^{\Phi}= & \sum_{k} \Phi_{k} \hat{y}_{i k} & Y_{i}^{\Theta \Theta}=\sum_{k} \Theta_{k}^{2} \hat{y}_{i k} \\
Y_{i}^{\Phi \Phi}= & \sum_{k} \Phi_{k}^{2} \hat{y}_{i k} \\
Y_{i}^{\Phi \Theta}= & \sum_{k} \Phi_{k} \Theta_{k} \hat{y}_{i k} . \\
M_{i} & =\left(\sum_{h} l_{i h} \phi_{h}^{\prime}\right)\left(Y_{i}^{\Phi \Phi} e_{i}+\frac{y_{i}\left(Y_{i}^{\Phi}\right)^{2}}{\hat{y}_{i}^{2}}\right) \\
& +\left(\sum_{h} l_{i h} \theta_{h}^{\prime}\right)\left(Y_{i}^{\Phi \Theta} e_{i}+\frac{y_{i} Y_{i}^{\Phi} Y_{i}^{\Theta}}{\hat{y}_{i}^{2}}\right) \\
N_{i} & =\left(\sum_{h} l_{i h} \phi_{h}^{\prime}\right)\left(Y_{i}^{\Phi \Theta} e_{i}+\frac{y_{i} Y_{i}^{\Phi} Y_{i}^{\Theta}}{\hat{y}_{i}^{2}}\right) \\
& +\left(\sum_{h} l_{i h} \theta_{h}^{\prime}\right)\left(Y_{i}^{\Theta \Theta} e_{i}+\frac{y_{i}\left(Y_{i}^{\Theta}\right)^{2}}{\hat{y}_{i}^{2}}\right)
\end{array}
$$

The complexity for each update is 8 (back)projections. As for MLTRC, a limited number of bins gives a sufficient representation of the spectrum.

Note that the condition that the Hessian of the likelihood should be negative definite, is only proved for MLTR without or with small scatter (if $\hat{y}_{i}^{2} \geq y_{i} s_{i}$ ), in other cases (substantial scatter, more complex model, ...) it cannot be proved. In our experience, this never caused convergence problems.

\section{Resolution}

The resolution is modeled by subsampling the detector elements ( $Q$ equally sized samples) and decreasing the pixel size in the reconstruction image $(M=J * f$, the new number of pixels with $f$ the (integer) size factor and $J$ the original number of pixels).

$$
\hat{y}_{i}=b_{i} \sum_{q=1}^{Q} \exp \left(-\sum_{m=1}^{M} l_{i q m} \mu_{m}\right)
$$

This resolution model does not take into account crosstalk and gantry motion.

\section{Scatter}

The influence of scatter is not always negligible. Including scatter, the projection model can be represented by $\hat{y}_{i}^{S}=\hat{y}_{i}+S p_{i}$, with $p_{i}$ a known profile and $S$ the profile factor. (This profile can be calculated e.g. by Monte Carlo simulations.) The profile factor $S$ will be updated in each iteration. The update strategy is the same as in (2), which gives:

$$
\begin{gathered}
\hat{y}_{i}^{S}=\hat{y}_{i}+S p_{i}, \quad L=\sum_{i}\left(y_{i} \ln \hat{y}_{i}^{S}-\hat{y}_{i}^{S}\right) \\
\Delta S=-\frac{\frac{\partial L}{\partial S}}{\frac{\partial^{2} L}{\partial S^{2}}} \\
=\frac{\sum_{i}\left(\frac{y_{i}}{\hat{y}_{i}^{S}}-1\right) p_{i}}{\sum_{i} \frac{y_{i}}{\left(\hat{y}_{i}^{S}\right)^{2}} p_{i}^{2}} .
\end{gathered}
$$

In this work, no scatter profile is available, a constant scatter profile, $p_{i}=1$, will be used.

\section{Local model reconstruction}

For the local model reconstruction, a proper projection model $\hat{y}_{i, p}$ will be assigned to each of the patches. As explained before, the models can differ in their energy and resolution properties. The combined projection estimate becomes:

$$
\hat{y}_{i}=\sum_{k} b_{i k} \prod_{p} z_{i k, p}+S p_{i} \text { with } \quad z_{i k, p}=\frac{\hat{y}_{i k, p}}{b_{i k}}
$$

Each $z_{i k, p}$ is calculated separately for each patch using the model assigned to that particular patch. During reconstruction, a patch will be treated as a group of pixels, or image-block, in a grouped coordinate algorithm ${ }^{23,25}$. This means that the patches are updated one by one, for each patch update only the voxels belonging to that particular patch are updated. Consequently, the sum over $h$ in the denominator of eq. (5) is also limited to these voxels. As will be discussed later, this has a significant effect on local convergence.

Note that the summation over the energies, $k$, happens after combining the projections of the different patches. Therefore it is difficult to combine true monochromatic and polychromatic energy models. IMPACT and MLTRC are both polychromatic algorithms, they both produce a projection for each energy bin $k$ and can therefore be combined in reconstructions where patches have different energy models. As mentioned before, using MLTRC instead of MLTR does not increase the computational complexity, moreover, cupping artifacts are reduced.

Updating the scatter term is a sinogram procedure which is applied after combining the contributions from different patches and different energy bins. Therefore eq. (18) will not be affected by the introduction of patches. 
All iterative reconstructions are initialized with a nonzero image. One could use the FBP reconstruction, but in our experience artifacts present in an initial reconstruction are often hard to remove. We prefer using a contour image. This is a contour of the object filled with the attenuation value of water. This contour image can of course be based on the FBP reconstruction. Here, the contour image will be based on the initial reconstruction which is also used to define the patches.

\section{Projection Completion}

The results obtained with the iterative methods will be compared to three projection completion methods. The methods start by making an initial FBP-reconstruction. Based on this image a segmentation of the metals, by $\mathrm{k}$-means clustering, is performed. The metal projections are determined by a forward projection of these segmented metals and removed from the original measurement. These projections are replaced by artificial projections. The new data set is then used to generate a new reconstruction with substantial artifact reduction.

The first PC method that will be used, is the classical PC as proposed by Kalender et al. ${ }^{1}$ where the metal projections are replaced by new ones based on linear interpolation. New streaks caused by removing information can (partly) by avoided by including a normalization step as in Müller et al. ${ }^{5}$ and Meyer et al. (NMAR) ${ }^{6}$. In this second approach (PC-NMAR), the measured sinogram is normalized, the interpolation step is applied and finally the interpolated sinogram is denormalized. For normalization a prior image is constructed. A segmentation of the original reconstruction defines air, tissue and bone regions. The prior image is built by assigning a particular attenuation value to the different regions. The air regions become $\mu_{j}=0(-1000 \mathrm{HU})$, the tissue region receives the typical attenuation value obtained at the particular energy settings of the scan (in HU this is around $0 \mathrm{HU}$ ). The bone regions retain their original values and the metal regions are set to $0 \mathrm{HU}$. The normalization is applied by dividing the measured sinogram by the projection of the prior image. Information about the intersection lengths for the different tissues is included, leading to reconstructions with less artifacts compared to regular PC. As mentioned in Meyer et al. ${ }^{6}$, when artifacts are severe, the segmentation process can be difficult and this can affect the prior image. Therefore, the prior image will be based on the result of a regular $\mathrm{PC}$ reconstruction. Note that even when using the normalized algorithm, artifacts can still be present because some information about the object is irreversibly lost during the replacement of the projections, e.g. edge information between different soft-tissue structures.

The third method, PC-FSMAR ${ }^{8}$, is an extension of the PC-NMAR method. The idea of this method is to retrieve edge information present in the FBP reconstruction by combining high frequencies of both FBP and PC-
NMAR reconstructions. PC-FSMAR results in the following weighted sum:

$\mu_{j}^{\text {FSMAR }}=\mu_{j}^{\text {NMARLow }}+w_{j} \mu_{j}^{\text {FBPHigh }}+\left(1-w_{j}\right) \mu_{j}^{\text {NMARHigh }}$

with $\mu_{j}^{\text {NMARLow }}$ the low frequencies of the PC-NMAR reconstruction, and $\mu_{j}^{\text {FBPHigh }}$ and $\mu_{j}^{\text {NMARHigh }}$ the high frequencies of the FBP and PC-NMAR reconstruction. The normalized weights $w_{j}$ decrease with increasing distance of pixel $j$ to the metal. An advantage of PC-FSMAR over other more advanced methods is the clear and rather straightforward implementation.

\section{SIMULATIONS AND MEASUREMENTS}

To evaluate the proposed techniques two phantoms were used. The first phantom is a circular phantom made of PMMA with diameter $19 \mathrm{~cm}$. It contains two aluminum inserts of $3 \mathrm{~cm}$ diameter and two iron inserts of $1 \mathrm{~cm}$ diameter as shown in figure 3 . The phantom structure is identical for the simulation and the measurement. The second phantom represents a part of a human pelvis and hips with two femoral implants. The phantom contains a titanium based implant and a cobalt-chromium based implant. An extra aluminum insert with diameter of $3 \mathrm{~cm}$ is added. Further some extra PMMA cylinders of $1 \mathrm{~cm}$ diameter are placed around the metals. This phantom was also simulated. Besides, a second composition of the body phantom was used for simulations. Here, the two implants remain and are now surrounded by a bone structure which is based on the bone structure in a human pelvis. The real and computer phantoms are depicted in figure 4 .

\section{A. Simulations}

The simulator is based on the CT-simulator of De Man et al. ${ }^{14}$ extended with a distance driven projector ${ }^{26}$. The simulations were two-dimensional and included Poisson noise, a polychromatic spectrum (50 bins) and subsampling of the detector elements (10 times), views ( 5 times), source ( 5 times, width $0.1 \mathrm{~cm}$ ) and image pixels of 0.01 $\mathrm{cm}$. The geometry and spectrum properties were based on a Siemens Sensation 16 system at $120 \mathrm{kV}$. The spectrum was determined using Spektr software ${ }^{27}$. The simulation also took into account afterglow and crosstalk between the detectors.

\section{B. Measurements}

Both phantoms were scanned on a Siemens Sensation 16 (part of a Biograph 16 PET/CT system). A circular scan at $120 \mathrm{kV}$ and $300 \mathrm{~mA}$ was performed using collimation $2 \times 1.00 \mathrm{~mm}$ and a rotation time of $0.5 \mathrm{~s}$. Only 

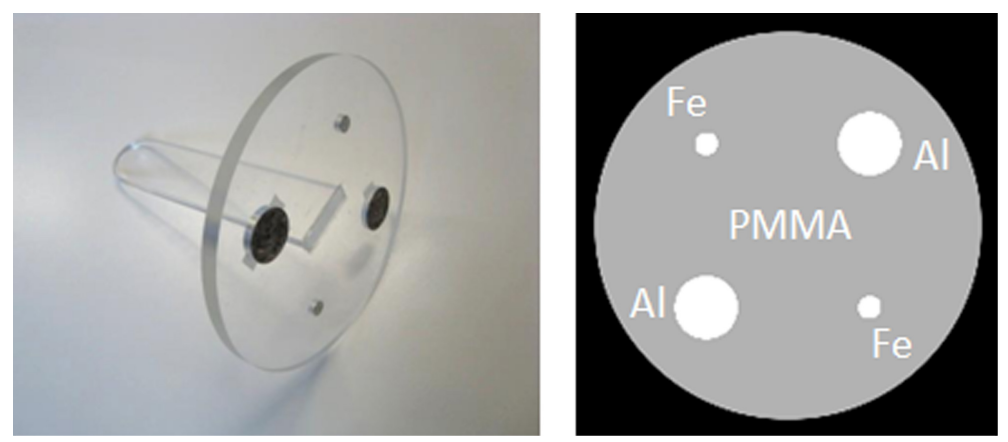

FIG. 3. Circular phantom The circular phantom on the left and its computer representation on the right. This phantom is a PMMA disk with diameter $19 \mathrm{~cm}$ and contains two cylindrical aluminum inserts of $3 \mathrm{~cm}$ diameter and two cylindrical iron inserts of $1 \mathrm{~cm}$ diameter.
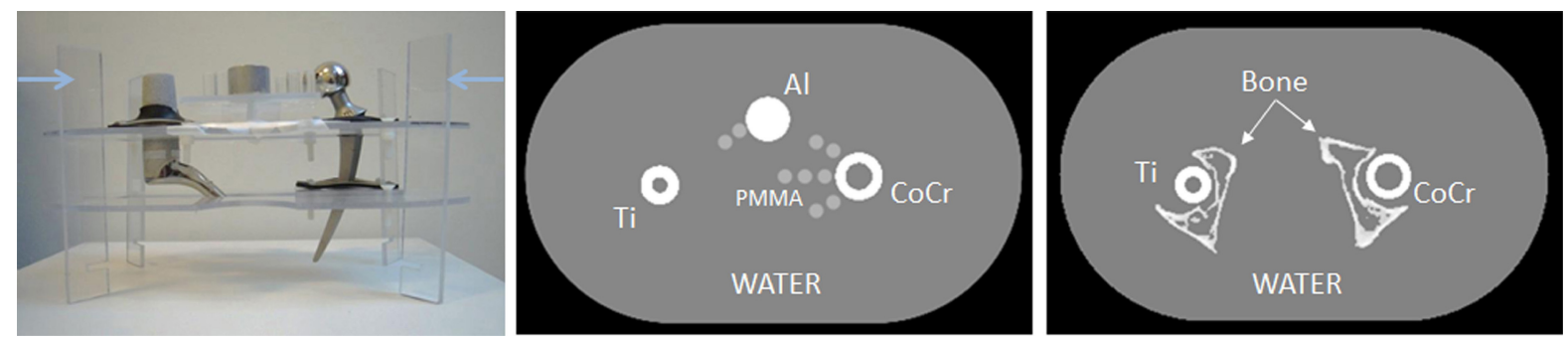

FIG. 4. Body phantom On the left the scaffold for the body phantom is shown. This scaffold is put into water for scanning. The slice which is scanned is marked with arrows. The computer representation of the slice is shown in the middle. This phantom contains two implants (titanium-based and cobalt-chromium-based), an aluminum insert with diameter $3 \mathrm{~cm}$ and several PMMA inserts of diameter $1 \mathrm{~cm}$. On the right, the variation of the computer phantom with a bone structure included, is also shown.

one slice of $1.00 \mathrm{~mm}$ will be used for a two-dimensional reconstruction.

Data obtained from clinical scanners are typically corrected for beam hardening and other effects. The corrections are often non-linear and can interfere with our proposed models. Siemens assisted to obtain raw data without beam hardening corrections.

\section{Reconstruction}

All data sets are reconstructed with different reconstruction schemes:

1. Four FBP-based reconstructions are performed: regular FBP, PC, PC-NMAR and PC-FSMAR.

2. A regular MLTR, MLTRC, and IMPACT reconstruction is done.

3. Different reconstructions based on a patchwork structure are performed:

(a) Patched MLTR, Patched MLTRC, or Patched IMPACT: Different patches are defined by the patched structure but the same energy model (MLTR, MLTRC or IMPACT) is used in all patches. (b) MLTRC + IMPACT: The energy model for the metal patches is IMPACT, the remaining patch without metals uses the MLTRC energy model.

(c) Resolution: For the patched methods, a second patched reconstruction has been done with increased resolution in the patches with the highest attenuating materials (iron and the implants). This means that three times more pixels were used and a threefold detector subsampling was applied for the projection of the particular patch.

Adaptive filtering of the projections ${ }^{28}$ is applied to all FBP-based reconstructions. The iterative reconstructions schemes consist of 20 iterations with 116 subsets.

\section{RESULTS}

\section{A. Simulations}

a. Circular computer phantom The circular (computer) phantom is reconstructed with a pixel size of 0.1 $\mathrm{cm}$. The results of the different reconstruction schemes are shown in figure 5 . 


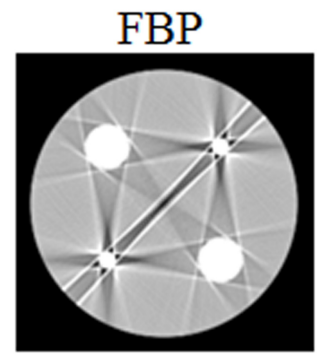

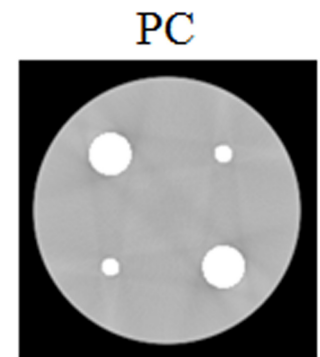

Regular
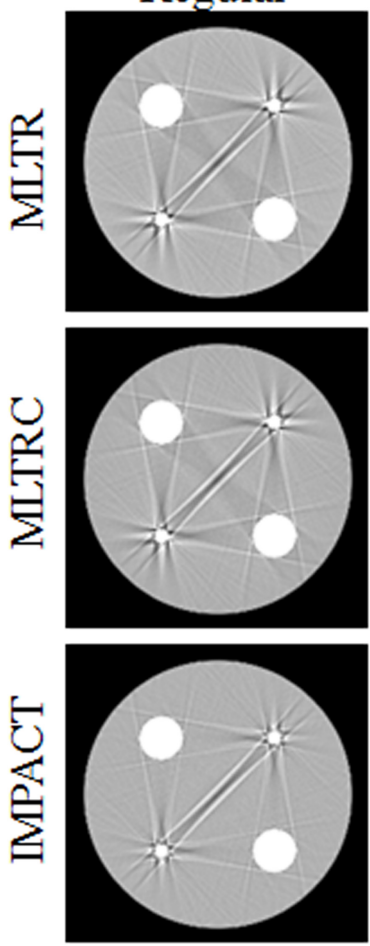

+
告
当

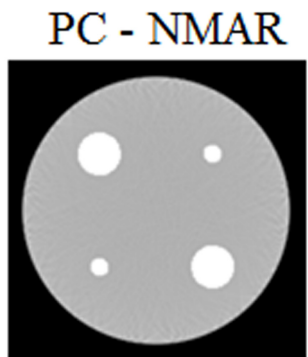

Patched
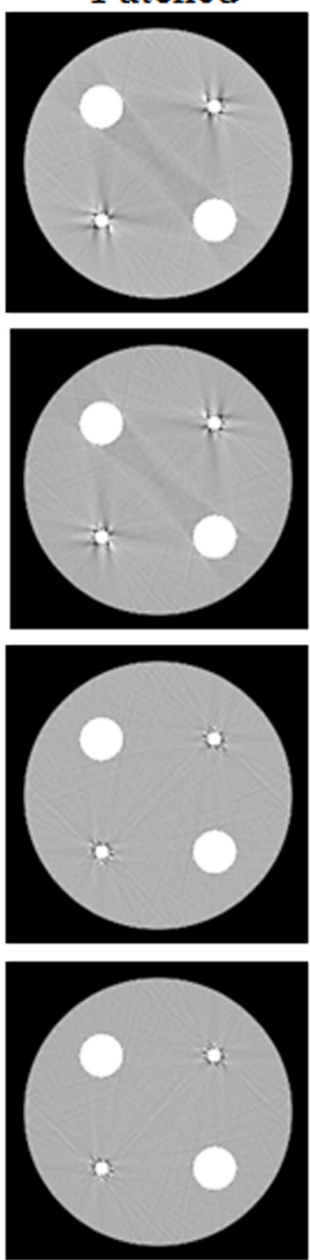

PC - FSMAR

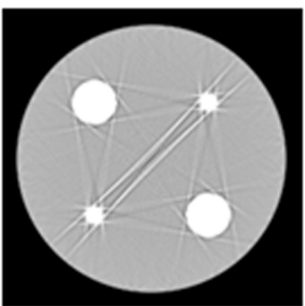

Patched + Resolution
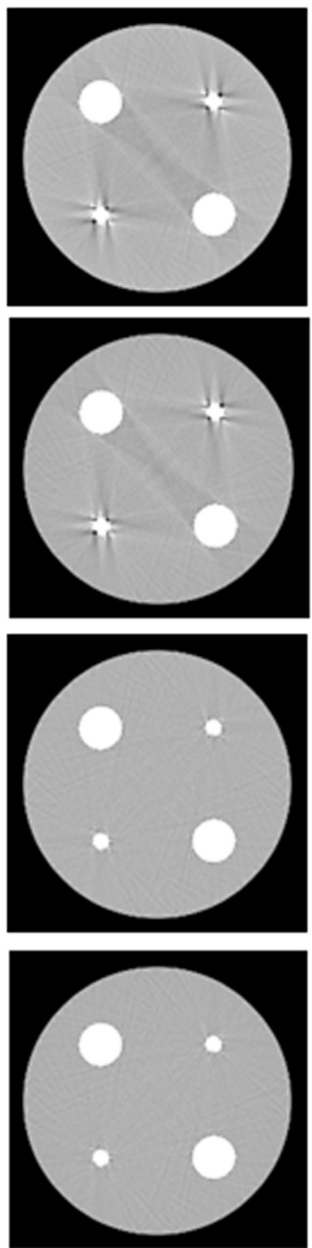

FIG. 5. Simulation, circular phantom Reconstruction of the circular (computer) phantom with FBP, PC, PC-NMAR, PC-FSMAR, MLTR, MLTRC, IMPACT, Patched MLTR, Patched MLTRC, Patched IMPACT and MLTRC+IMPACT. When using patches a reconstruction with and without resolution modeling in the Fe-patches is show. (Window $=750 \mathrm{HU}, \mathrm{Center}=$ 0 HU.) 
Artifacts are reduced for MLTR, MLTRC, and IMPACT compared to FBP. Differences between MLTR, MLTRC, and IMPACT are small. In a narrow window, MLTRC and IMPACT show reduced cupping compared to MLTR, and the IMPACT reconstruction has no dark streak between the aluminum inserts. The difference between the reconstruction methods becomes more clear when using a patched update, with IMPACT having no streaks in between any of the metallic inserts. Introducing a higher resolution for the iron patches has some influence but very little. Note that MLTRC+IMPACT, using MLTRC for the non-metal patches, gives a very similar result as using IMPACT for the whole image.

The results for PC and PC-NMAR are almost perfect. However, this is an almost ideal situation: a symmetrical homogeneous circular phantom. PC-NMAR is performing better that regular PC. PC-FSMAR reintroduces some of FBP artefacts in the $\mathrm{PC}$ reconstruction.

b. Body computer phantom

Body phantom with PMMA details The pixel size for this reconstruction was $0.1 \mathrm{~cm}$. The overall results for the body phantom (fig. 6) are similar to the results of the circular phantom. The cobaltchromium femoral prosthesis is attenuating more and consequently suffers more from beam hardening, making MLTR worse compared to MLTRC and IMPACT. Also here, the patched reconstruction methods perform better that the regular reconstruction methods. Using MLTRC+IMPACT results in the same image quality as Patched IMPACT.

PC, and especially PC-NMAR, give a smooth, seemingly artifact-free result. However, the PMMA details are deformed or have disappeared. These details are partly recovered by PC-FSMAR. However, together with high frequency information from the object, also some of the streaks and shadows from the FBP image are reintroduced and cover some PMMA elements.

Body phantom with bone structure The reconstruction pixel size is $0.1 \mathrm{~cm}$. Figure 7 shows the results for FBP, PC-NMAR, PC-FSMAR, IMPACT, Patched IMPACT and MLTRC+IMPACT. The results for the other methods are not shown, but they are analogous to previous examples. The edges of the bone structure are not sufficiently reconstructed with PC or PC-NMAR. PC-FSMAR regains some contrast, but also here FBP artifacts are retrieved together with edge information. The iterative methods are reconstructing the bone structure well. Note that bone is segmented into the major patch which is reconstructed with MLTRC in the combined MLTRC+IMPACT reconstruction, showing that MLTRC suffices for the reconstruction of these bone regions.

\section{B. Measurements}

c. Circular phantom The measurement was reconstructed on a grid with isotropic pixels of $0.098 \mathrm{~cm}$. The results for the measurement of the circular phantom (fig. 8) are similar to those for the simulated data set. The introduction of patches in Patched IMPACT leads to a substantial artifact reduction compared to regular IMPACT. Replacing IMPACT by MLTRC in the non-metal patch does not lead to image degradation. The results for projection completion are equivalent to the simulations.

d. Body phantom The measurement of the body phantom was reconstructed on a grid with isotropic pixels of $0.098 \mathrm{~cm}$. The results are shown in figure 9 . They are comparable to those of the simulation. With Patched IMPACT and MLTRC + IMPACT strongly reducing the artifacts and only little influence of the use of an increased resolution for the implant patches. The details around the implants are reconstructed well. The results for PC and PC-NMAR have only mild streaks or shadows with PC-NMAR outperforming PC. The PMMA details are deformed for PC and PC-NMAR and, as in the simulation, partly retrieved in PC-FSMAR.

\section{v. DISCUSSION}

Two important methods for metal artifact reduction are projection completion and iterative reconstruction with advanced projection model. Where projection completion is fast, the influence of replacing data always includes a risk. Iterative reconstruction uses all available information but is extremely slow when using a more complex model. In this study we presented a local model reconstruction scheme where complex models are only applied to a limited area of the reconstruction volume. The framework for our local model scheme is the patchwork structure and projector.

The reconstruction volume was divided in several regions or patches. For each of these patches a different energy and resolution model could be defined. This is very similar to the region of interest reconstruction proposed by Hamelin et al. ${ }^{29,30}$ and Yu et al. ${ }^{31}$. In these papers, the approach was used to obtain a high resolution reconstruction in a small region of interest, in combination with a coarser (and faster) reconstruction of the surrounding background. Here, this approach was extended to implement a locally improved energy model and improved sampling of the detector elements. The patches are updated sequentially, each patch was considered as a group of pixels in a grouped coordinate algorithm. In a first test, the patched reconstruction was applied. Here, the volume is divided into the different patches, but the same model was used for all patches. In all examples, the application of the sequential patch update already reduced the artifacts, especially the streaks connecting the metals. The origin of this result is an improved convergence for the metal patches. Sequentially updating groups of pixels is known to improve convergence ${ }^{23,32}$. The denominator of the update steps will be smaller 


\section{FBP}
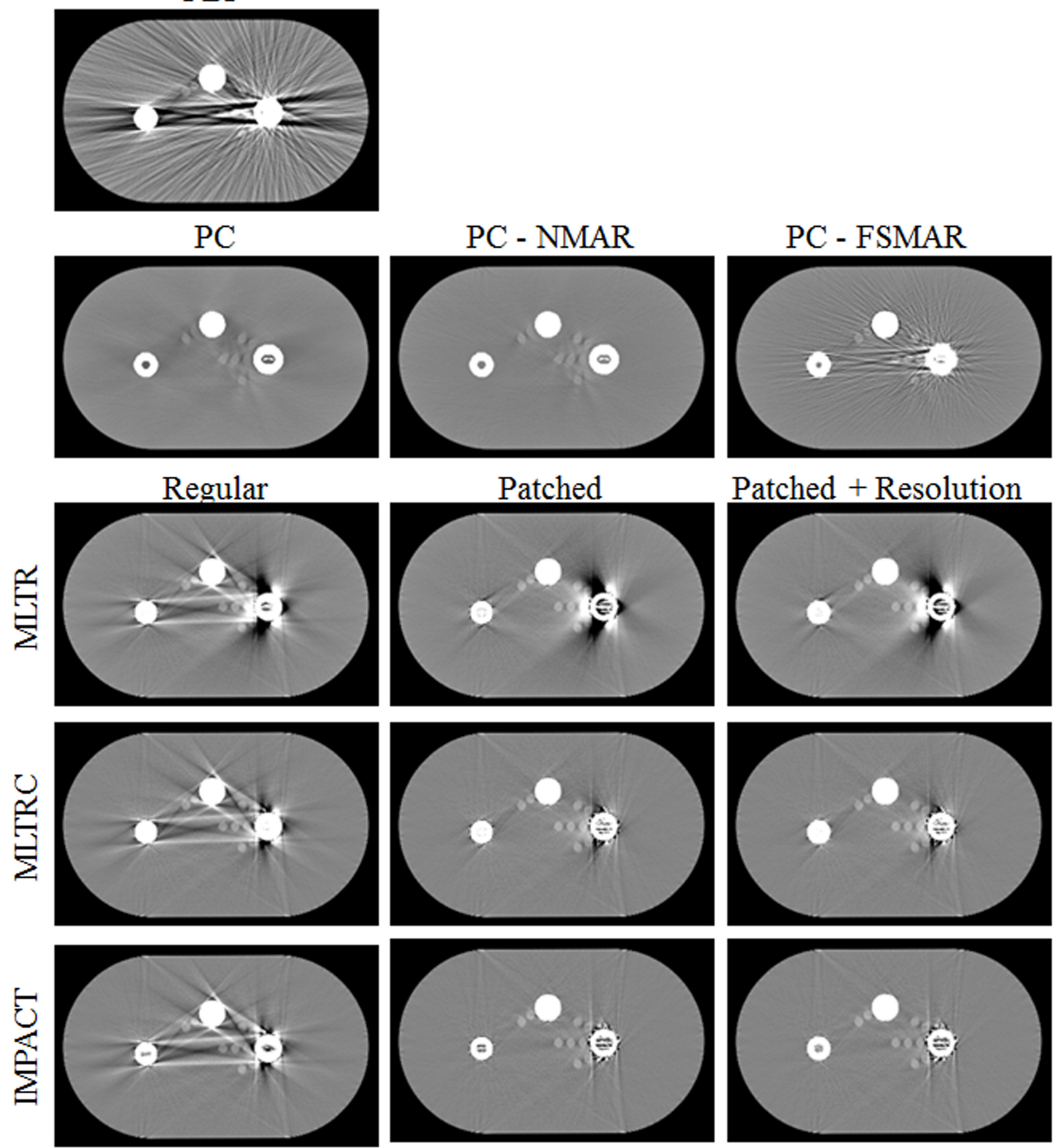

兒㫐
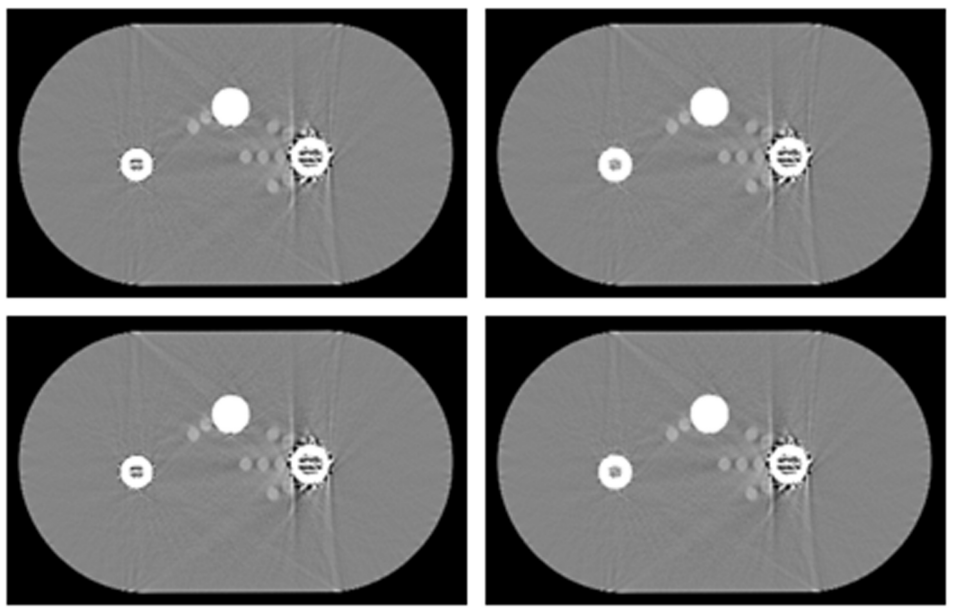

FIG. 6. Simulation, body phantom with PMMA details Reconstruction of the body (computer) phantom with PMMA elements. The methods are FBP, PC, PC-NMAR, PC-FSMAR, MLTR, MLTRC, IMPACT, Patched MLTR, Patched MLTRC, Patched IMPACT and MLTRC+IMPACT. A reconstruction with and without resolution modeling in the implant-patches is shown. (Window $=750 \mathrm{HU}$, Center $=0 \mathrm{HU}$.) 


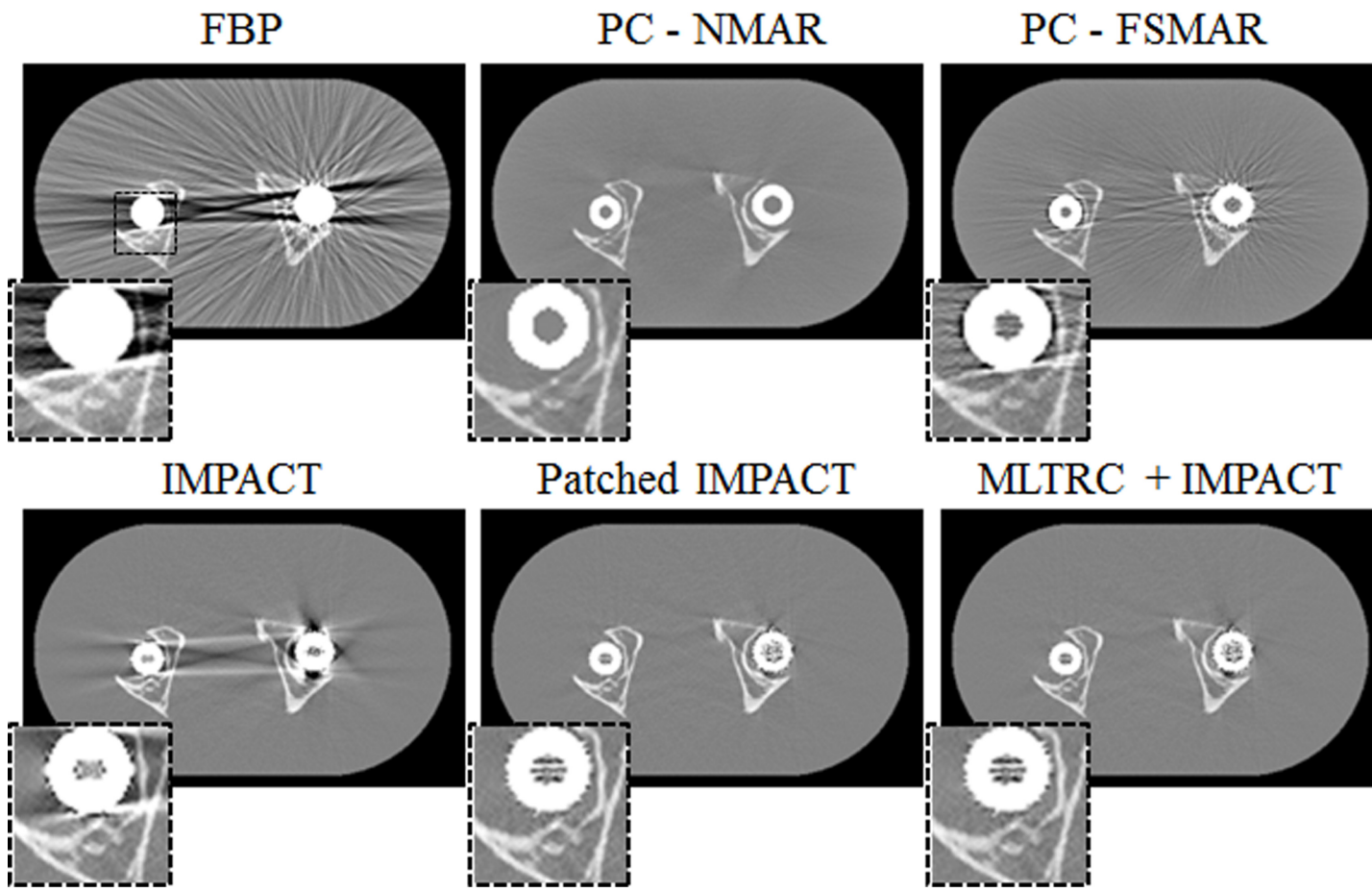

FIG. 7. Simulation, body phantom with bone Reconstruction of the body (computer) phantom with bone. The methods are FBP, PC-NMAR, PC-FSMAR, IMPACT, Patched IMPACT and MLTRC+IMPACT (no resolution modeling). The region around the left implant is magnified in the inserts. (Window $=750 \mathrm{HU}$, Center $=0 \mathrm{HU}$.)

when the area of the updated patch is smaller. For instance, in the MLTR update step (7), the sum $\sum_{h} l_{i h}$ in the denominator is proportional to the width of the updated patch. A smaller denominator makes the update larger. By giving the metal patches a larger update compared to the rest of the image, the relative convergence is altered which results in higher attenuation values and sharper edges in an earlier stage of the reconstruction process, resulting in less artifacts. A simulation experiment not shown here confirmed that for far more iterations (about 200 iterations x 116 subsets) the nonpatched and patched reconstruction converge to a very similar solution. A similar reduction of streaks is obtained but both reconstructions suffer from fine streak artifacts caused by the well known noise characteristics. In $\mathrm{Yu}$ et $\mathrm{al}^{33}$ is also shown that altering the convergence of different parts of the image, such as edges, can result in an improved image quality.

We hypothesized that the non-metal regions could be reconstructed with a less complex model. Therefore, we combined the energy model of MLTRC for the non-metal patch with IMPACT for the metal patches. In all examples this resulted in a very similar artifact reduction compared to Patched IMPACT. The same image quality was achieved while reducing the complexity of the largest part of the reconstruction volume from 8 (back)projections for IMPACT to 3 (back)projections for MLTRC. Artifacts originating from non-linear partial volume effects could be reduced by increasing the resolution. However, for all phantoms, the influence of an increased resolution was rather low. It is questionable whether this small improvement is worth the extra time.

As a comparison, three projection completion methods were applied to the same data sets. Regular PC and PC-NMAR reconstructions have less obvious artifacts compared to the iterative methods which all suffered from remaining (but reduced) shadow and streak artifacts. However, structures around the metals are often poorly reconstructed when using PC or PC-NMAR. PC-FSMAR tries to solve this problem by reintroducing some high frequency information from the initial FBP reconstruction. Part of the edge information is indeed retrieved, but in the phantom with the bone structure, the bone is still less sharp compared to the iterative reconstructions. Unfortunately, PC-FSMAR is not free of streaks or shadows like the other projection completion methods because some artifacts are reintroduced by using the high frequency information of the FBP reconstruction. The loss of contrast and edges, especially close to the implants, can be important for some application, like implant follow-up in orthopedic surgery. Other advanced projection completions methods ${ }^{4,7,9-12}$ could be more successful in retrieving contrast, although by replacing projection values some contrast information might still be lost.

The reconstructions with Patched IMPACT or MLTRC+IMPACT still contain artifacts: fine streaks around the metals, small brighter dots, darker regions in 


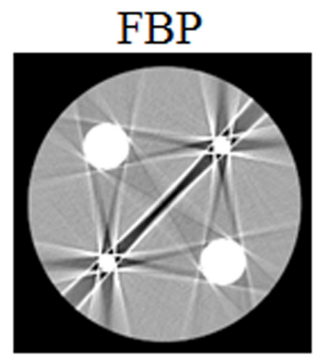

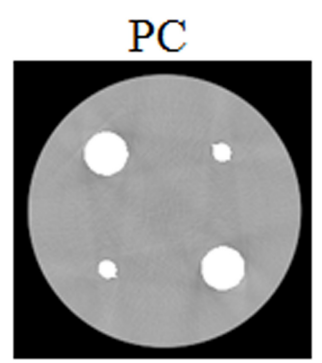
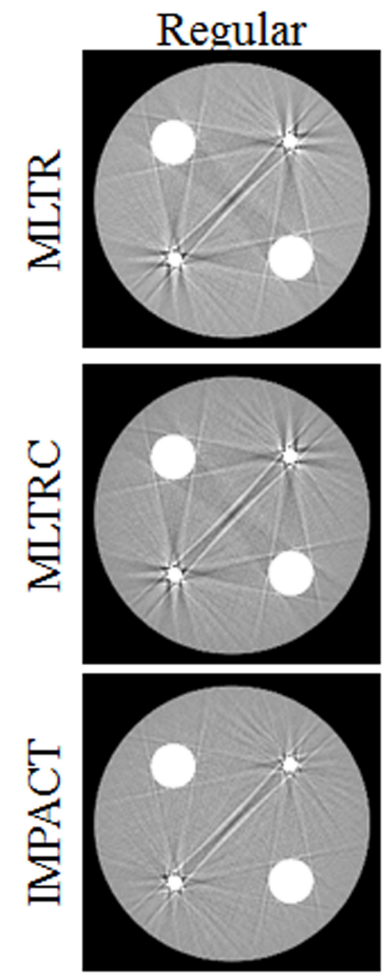

+
当
$\sum$
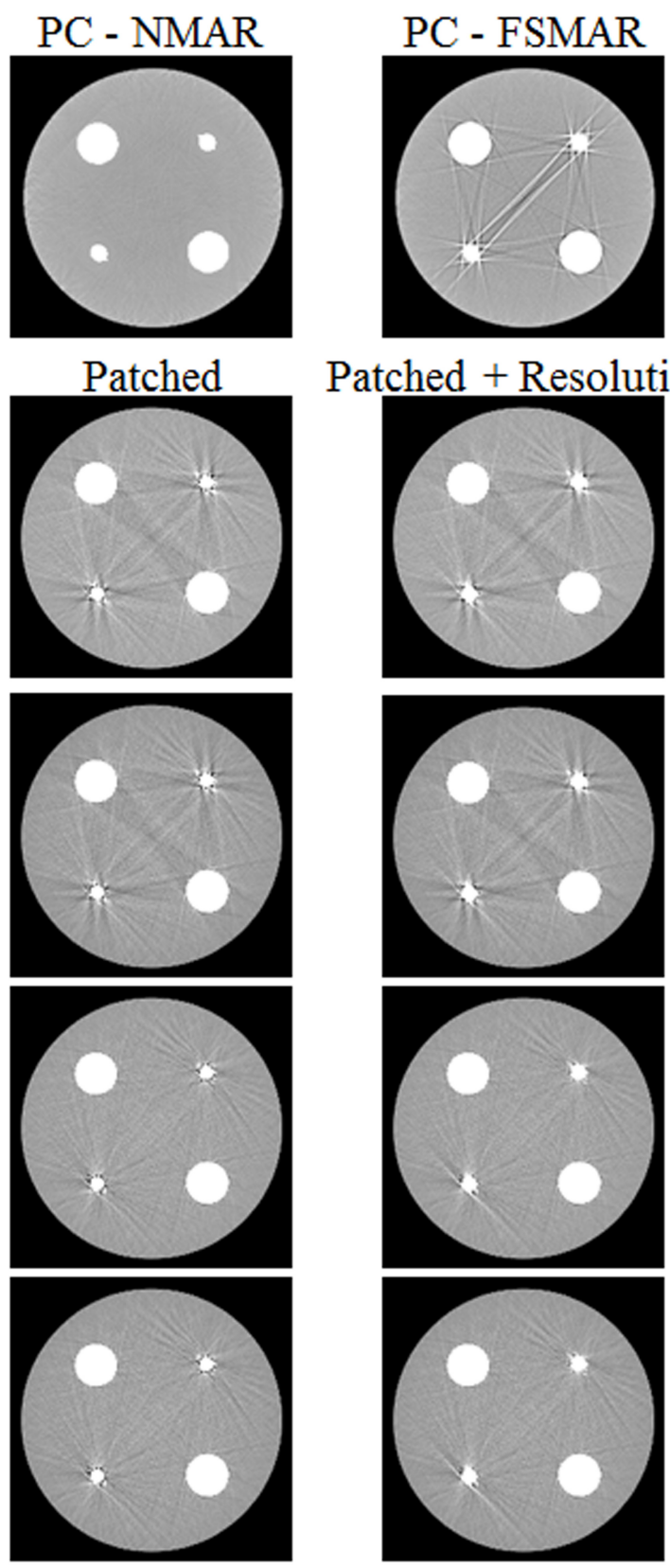

Patched + Resolution
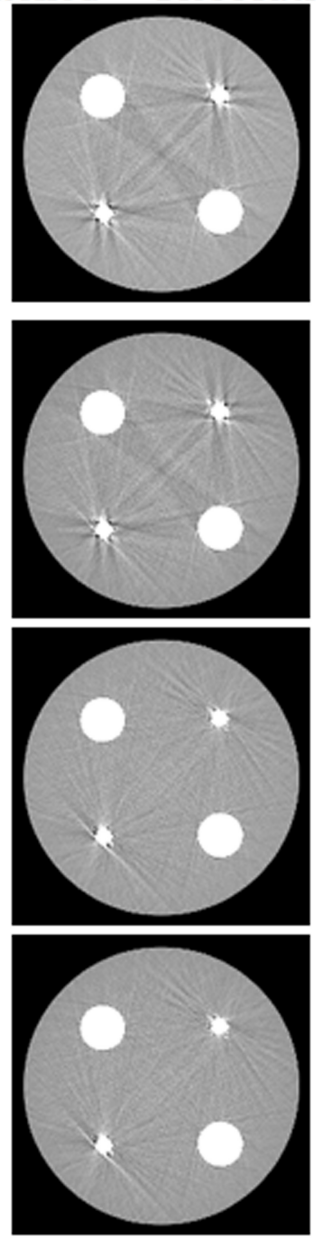

FIG. 8. Measurement, circular phantom Reconstruction of the circular phantom with FBP, PC, PC-NMAR, PC-FSMAR, MLTR, MLTRC, IMPACT, Patched MLTR, Patched MLTRC, Patched IMPACT and MLTRC+IMPACT. When using patches a reconstruction with and without resolution modeling in the Fe-patches is show. (Window $=750 \mathrm{HU}$, Center $=0 \mathrm{HU}$.) 


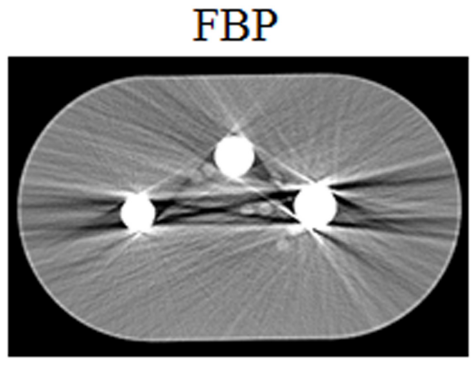

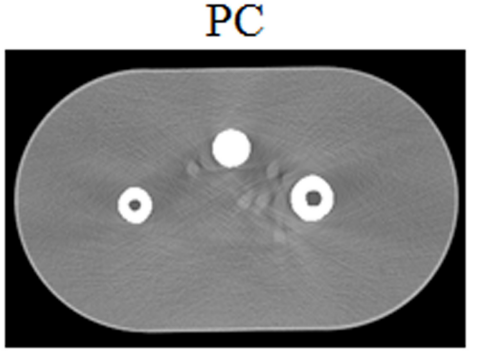
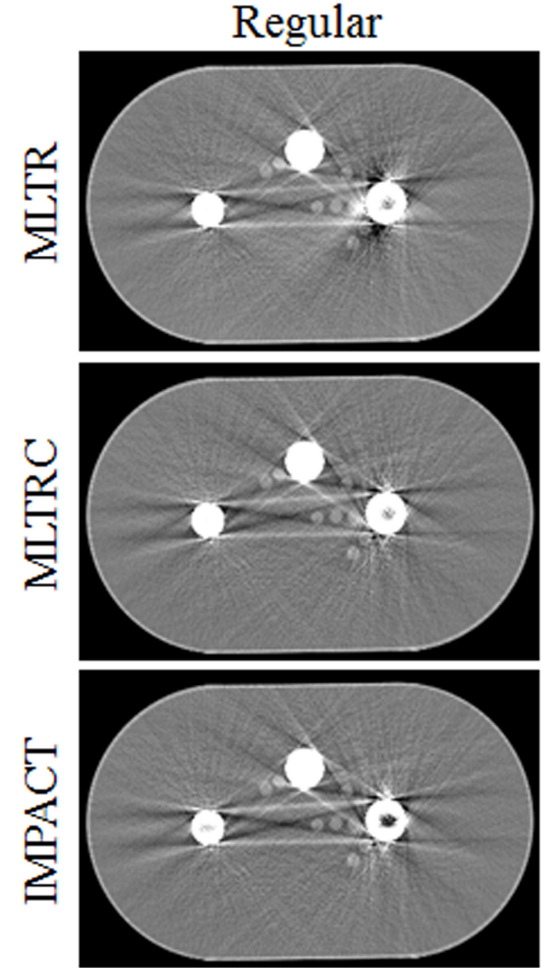

䓠
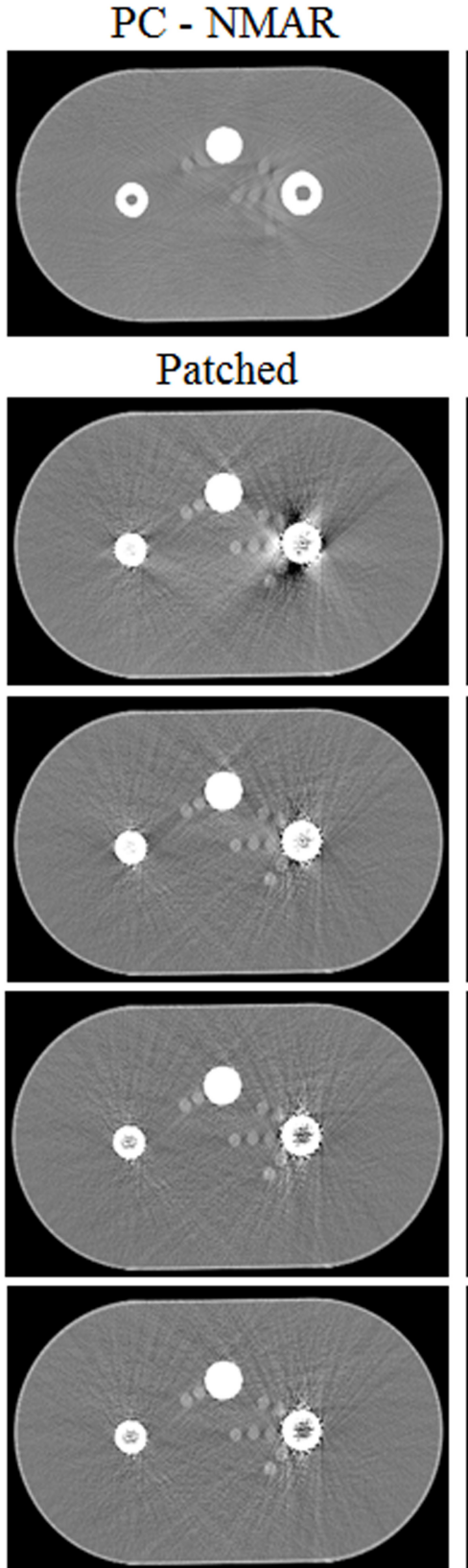

PC - FSMAR

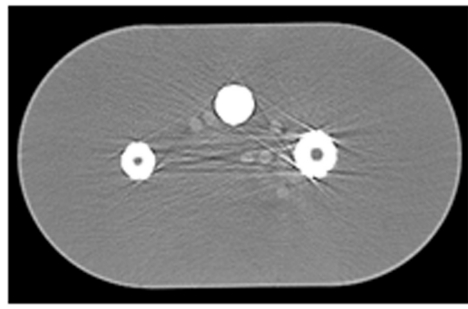

Patched + Resolution
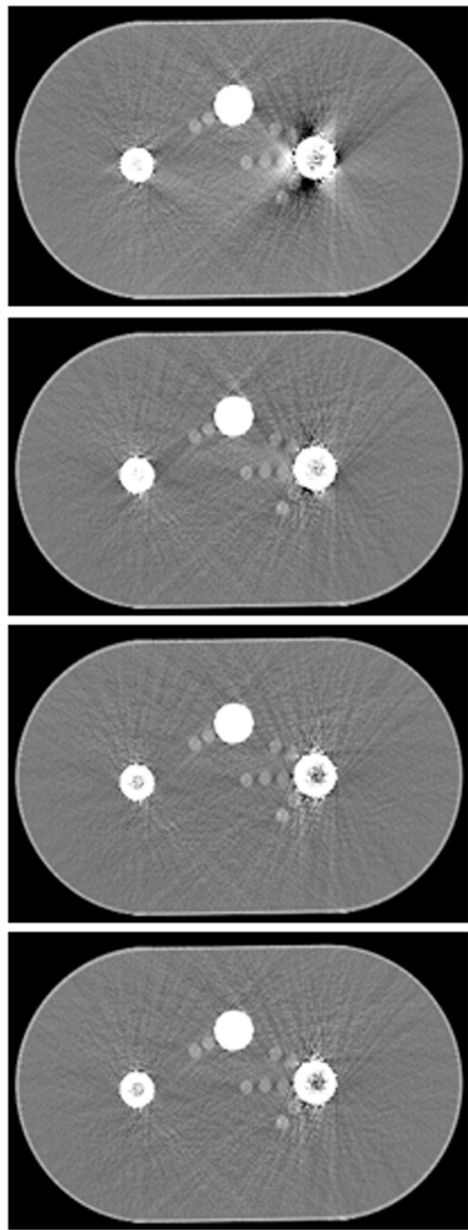

FIG. 9. Measurement, body phantom Reconstruction of the body phantom with FBP, PC, PC-NMAR, PC-FSMAR, MLTR, MLTRC, IMPACT, Patched MLTR, Patched MLTRC, Patched IMPACT and MLTRC+IMPACT. A reconstruction with and without resolution modeling in the implant-patches is shown. (Window $=750 \mathrm{HU}$, Center $=0 \mathrm{HU}$.) 
between the metals. The origin of fine streaks and dots is probably noise. Further, although iterative reconstruction is using all available information, some important information can be lost during the measurement itself, as for instance in case of photon starvation. The remaining artifacts could be reduced by introducing Markov priors in the reconstruction algorithm ${ }^{34}$. Notice that when using priors, the properties of the prior could also be changed depending on the particular patch. A second origin of artifacts might be scatter. We believe the scatter in the examples shown is relatively low and it is sufficient to use a simple scatter model, a constant factor. Depending on the system geometry and the object structure and attenuation, a more accurate (Monte Carlo simulated) scatter profile could be necessary to sufficiently reduce image artifacts.

In this study we chose to use thresholding to select the metals in the initial reconstruction. In projection completion, mistakes in the metal segmentation will affect the final reconstruction. In contrast, the segmentation used for the patch definition does not determine the shape or voxel values of the metals. The advantage of using a more sophisticated segmentation method will thus be small. Selecting a region larger than the metal will not affect the final artifact reduction. Selecting a region smaller than the metal, which can be avoided in many cases, could result in a less efficient artifact reduction because the model will not be applied to the entire metal region. To avoid selecting regions that are smaller than the metal, the metal regions were dilated after thresholding.

In this study we used local models for reconstruction with artifact reduction. The patchwork structure as presented here can also be used for other than MAR applications, e.g. reconstruction with varying resolution over the image ${ }^{29-31,35-37}$, and for other modalities than CT, e.g. PET or SPECT.

\section{CONCLUSION}

Projection completion methods are fast and efficient methods for metal artifact reduction but they tend to deform structures lying in the interpolated projection rays. Iterative reconstructions have some remaining streaks and shadows, although strongly reduced, but by using all available information a better reconstruction of structures close to and in between metals is obtained. The increased computation time caused by using more complex models can be limited by applying the more sophisticated model only locally in and around the metals. This local model reconstruction does not result in loss of image quality. Moreover, sequentially updating the different image parts leads to an improved artifact reduction.

\section{ACKNOWLEDGMENTS}

The authors would like to thank dr. Karl Stierstorfer from Siemens AG, Healthcare Sector, Forchheim, Germany, for his valuable suggestions and help with the data format and preprocessing and prof. dr. Michiel Mulier for the femoral implants and for the interesting discussion on the problems with implant imaging for orthopedic surgery.

This work is supported by the SBO-project QUANTIVIAM (060819) of the Institute for the Promotion of Innovation through Science and Technology in Flanders (IWT-Vlaanderen).

${ }^{1}$ W. Kalender, R. Hebel and J. Ebersberger, "Reduction of CT artifacts caused by metallic implants," Radiology 164, 567-568 (1987).

${ }^{2}$ G. Glover and N. Pelc, "An algorithm for the reduction of metal clip artifacts in CT reconstructions," Med. Phys. 8(6), 799-807 (1981).

${ }^{3}$ A. Mahnken, R. Raupach, J.E. Wildberger, B. Jung, N. Heussen, T.G. Flohr, R.W. Günther and S. Schaller, "A new algorithm for metal artifact reduction in computed tomography, in vitro and in vivo evaluation after total hip replacement." Invest. Radiol. 38(12), 769-775 (2003).

${ }^{4}$ C. Lemmens, D. Faul and J. Nuyts, "Suppression of metal artifacts in CT using a reconstruction procedure that combines MAP and projection completion," IEEE Trans. Med. Imag. 28(2), 250260 (2009).

${ }^{5}$ J. Müller and T.M. Buzug, "Spurious structures created by interpolation-based CT metal artifact reduction," Proc. of SPIE 7258(1), 1Y1-1Y8 (2009).

${ }^{6}$ E. Meyer, R. Raupach, M. Lell, B. Schmidt and M. Kachelrieß, "Normalized metal artifact reduction (NMAR) in computed tomography," Med. Phys. 37(10), 5462-5493 (2010).

${ }^{7}$ M. Bal and L. Spies, "Metal artifact reduction in CT using tissueclass modeling and adaptive prefiltering," Med. Phys. 33(8), 2852-2559 (2006)

${ }^{8}$ E. Meyer, R. Raupach, M. Lell, B. Schmidt and M. Kachelrieß, "Frequency split metal artifact reduction in computed tomography," Med. Phys. 39(4), 1940-1916 (2012).

${ }^{9}$ O. Watzke and W.A. Kalender, "A pragmatic approach to metal artifact reduction in CT: merging of metal artifact reduced images," Eur. Radiol. 14, 849-856 (2014).

${ }^{10}$ E. Meyer, R. Raupach, B. Schmidt, A.H. Mahnken and M. Kachelrieß, "Adaptive normalized metal artifact reduction (ANMAR) in computed tomography," IEEE Nucl. Sci. Sym. Conf. Rec., Valencia, Spain, 2560-2565 (2011).

${ }^{11}$ S. Tohnak, A.J.H. Mehnert, M. Mahoney and S. Crozier, "Dental CT metal artefact reduction based on sequential substitution," Dentomaxillofacial Radiology 40, 184-190 (2011).

${ }^{12}$ M. Yazdi, M.A. Lari, G. Bernier and L. Beaulieu, "An opposite view data replacement approach for reducing artifacts due to metallic dental objects," Med. Phys. 38(4), 2275-2281 (2011).

${ }^{13}$ J.F. Barrett and N. Keat, "Artifacts in CT: Recognition and avoidance," RadioGraphics 24, 1679-1691 (2004).

${ }^{14}$ B. De Man, J. Nuyts, P. Dupont, G. Marchal and P. Suetens, "Metal streak artifacts in X-ray computed tomography: a simulation study," IEEE Trans. Nucl. Sci. 46(3), 691-696 (1999).

${ }^{15}$ J. Williamson, B. Whiting, J. Benac, R. Murphy, G. Blaine, J. O'Sullivan, D. Politte and D. Snyder, "Prospects for quantitative computed tomography imaging in the presence of foreign metal bodies using statistical image reconstruction," Med. Phys. 29 (10), 2404-2418 (2002)

${ }^{16}$ B. De Man, J. Nuyts, P. Dupont, G. Marchal and P. Suetens, "Iterative maximum-likelihood polychromatic algorithm for CT," IEEE Trans. Med. Imag. 14(1), 132-137 (1995). 
${ }^{17}$ I. Elbakri and J. Fessler, "Statistical image reconstruction for polyenergetic X-ray computed tomography," IEEE Trans. Med. Imag. 21(2), 89-99 (2002).

${ }^{18}$ N. Menvielle, Y. Goussard, D. Orban and G. Soulez, "Reduction of beam-hardening artifacts in X-ray CT," IEEE EMB Conference Proceedings, Shanghai, China, 1865-1868 (2005).

${ }^{19}$ I. Elbakri and J. Fessler, "Segmentation-free statistical image reconstruction for polyenergetic X-ray computed tomography with experimental validation," Phys. Med. Biol. 48, 2453-2477 (2003).

${ }^{20} \mathrm{~B}$. De Man, Iterative reconstruction for reduction of metal artifacts in computed tomography. (Katholieke Universiteit Leuven, Belgium, 2001), PhD dissertation.

${ }^{21}$ W. Zbijewski and F.J. Beekman , "Characterization and suppression of the edge and aliasing artifacts in iterative x-ray CT reconstruction," Phys. Med. Biol. 49, 145-157 (2004).

${ }^{22}$ J. Nuyts, B. De Man, P. Dupont, M. Defrise, P. Suetens and L. Mortelmans, "Iterative reconstruction for helical CT: a simulation study," Phys. Med. Biol. 43, 729-737 (1998).

${ }^{23}$ J.A. Fessler, E.P. Picare, N.H. Clinthorne and K. Lange, "Grouped-coordinate ascent algorithm for penalized-likelihood transmission image reconstruction," IEEE Trans. Med. Imag. 16(2), 166-175 (1997).

${ }^{24} \mathrm{G}$. Herman, "Correction for beam hardening in computed tomography," Phys. Med. Biol. 24(1), 81-106 (1979).

${ }^{25}$ J.A. Fessler and K. Donghwan, "Axial block coordinate descent (ABCD) algorithm for X-ray CT image reconstruction," Proc. Intl. Mtg. on Fully 3D image Recon. in Rad. and Nuc. Med., Potsdam, Germany, 262-265 (2011).

${ }^{26}$ B. De Man and S. Basu, "Distance-driven projection and backprojection in three dimensions," Phys. Med. Biol. 49, 2463-2475 (2004).

${ }^{27}$ J.H. Siewerdsen, A.M. Waese, D. J. Moseley, S. Richard and D. A. Jaffray," "Spektr: A computational tool for x-ray spectral analysis and imaging system optimization," Med. Phys. 3(1), 3057-3067 (2004).

${ }^{28}$ M. Kachelrieß, O. Watzke and W. A. Kalender, "Generalized multi-dimensional adaptive filtering for convectional and spiral single-slice, multi-slice, and cone-beam CT," Med. Phys. 28(4), 475-490 (2001).

${ }^{29}$ B. Hamelin, Y. Goussard, J. Dussault, G. Cloutier, G. Beaudoin and G. Soulez, "Design of iterative ROI transmission tomography reconstruction procedures and image quality analysis," Med. Phys. 37(9), 4577-4589 (2010).

${ }^{30}$ B. Hamelin, Y. Goussard, D. Gendron, J. Dussault, G. Cloutier, G. Beaudoin and G. Soulez, "Iterative reconstruction of real data with metal artifact reduction," IEEE Int. Symp. on Bio. Im., Paris, France, 1453-1456 (2008).

${ }^{31}$ Z. Yu, J. Thibault, C.A. Bouman, K.D. Sauer and J. Hsieh, "Edge-localized iterative reconstruction for computed tomography," Proc. Intl. Mtg. on Fully 3D image Recon. in Rad. and Nuc. Med., Beijing, China, 255-258 (2009).

${ }^{32}$ K. Van Slambrouck and J. Nuyts, "Accelerated convergence with image-block iterative reconstruction," IEEE Nucl. Sci. Sym. Conf. Rec., Valencia, Spain, 2576-2580 (2011).

${ }^{33}$ Z. Yu, J. Thibault, C.A. Bouman, K.D. Sauer and J. Hsieh, "Fast model-based X-ray CT reconstruction using spatially nonhomogeneous ICD optimization," IEEE Trans. Im. Pro. 20(1), 161-175 (2011).

${ }^{34}$ B. De Man, J. Nuyts, P. Dupont, G. Marchal, P. Suetens, "Reduction of metal streak artefacts in x-ray computed tomography using a transmission maximum a posteriori algorithm," IEEE Trans. Nucl. Sci. 47 (3), 977-981 (2000)

${ }^{35}$ L.J. Meng and N. Li, "Non-uniform object-space pixelation (NUOP) for penalized maximum-likelihood image reconstruction for a single photon emission microscope system," IEEE Trans. Nucl. Sci. 56 (5), 2777-2788 (2009).

${ }^{36}$ J.S. Maltz, "Optimal time-activity basis selection for exponential spectral analysis: application to the solution of large dynamic emission tomographic reconstruction problems," IEEE Trans. Nucl. Sc. 49(4), 1452-1464 (2001).

${ }^{37}$ J.G. Brankov and M.N. Wernick, "Tomographic image reconstruction using content-adaptive mesh modeling," Proc. IEEE Intl. Conf. on Image Processing, Thessaloniki , Greece, 690-693 (2001). 